\title{
MELHORAMENTO ANIMAL: PREDIÇÃO DE VALORES GENÉTICOS PELO MODELO ANIMAL - BLUP EM BOVINOS DE LEITE, BOVINOS DE CORTE, OVINOS E SUÍNOS
}

\author{
MARCOS DEON VILLELA DE RESENDE ${ }^{1}$; JESUS ROLANDO H. ROSA PEREZ
}

Departamento de Zootecnia, Setor de Ciências Agrárias, Universidade Federal do Paraná.

\begin{abstract}
Animal model - BLUP is the standard procedure for animals genetic evaluation. This paper deals with practical aspects concerning the application of this procedure for the improvement of the main species of domestic animals. Several animal models and measures associated to the predicted breeding values and accuracy of predictions are considered.
\end{abstract}

RESUMO - Atualmente, o procedimento padrão de avaliação genética é o BLUP sob modelo animal. O presente trabalho apresenta aspectos práticos relativos à aplicação deste procedimento no melhoramento das principais espécies de animais domésticos. São abordados vários modelos animais e as várias medidas associadas aos valores genéticos preditos e à precisão das predições.

\section{Introdução}

O sucesso do melhoramento genético animal depende, essencialmente, da adoção de precisos métodos de seleção, os quais demandam a predição dos valores genéticos dos animais candidatos à seleção. O procedimento de predição de valores genéticos denominado BLUP (melhor predição linear não viciada) foi desenvolvido por HENDERSON em 1949 e apresentado formalmente em 1973 (HENDERSON, 1973; 1984). A partir de 1990, com o desenvolvimento da tecnologia computacional, o BLUP associado ao modelo animal tornouse $o$ procedimento padrão de avaliação genética em bovinos de leite, bovinos de corte, ovinos e suínos. Dessa forma, atualmente, torna-se fundamental o conhecimento dos princípios e interpretações associados à aplicação do modelo animal no melhoramento animal, seja por parte de produtores, técnicos, estudantes e professores.

Com base no exposto, o presente trabalho objetiva contribuir no processo de difusão de conhecimentos associados ao uso do modelo animal em programas práticos de melhoramento genético animal.

\section{Modelo Animal - BLUP}

As observações (medições) realizadas sobre determinada característica, como por exemplo a produção de leite, podem ser explicadas pelo seguinte modelo, denominado modelo animal:

$\mathrm{Y}=\mathrm{X} \beta+\mathrm{Z} \mathrm{a}+\mathrm{e}$, onde:

$\mathrm{Y}$ - vetor dos dados observados nos vários (n) animais, de dimensão $\mathrm{n} \times 1$;

$\beta$ - vetor contendo efeitos ambientais identificáveis

\footnotetext{
${ }^{1}$ Pesquisador da EMBRAPA.
}

ou efeitos fixos do modelo, como por exemplo os efeitos de sexo, de grupo de manejo, de grupo contemporâneo, dentre outros. Este vetor tem dimensão $\mathrm{p} \times 1$, onde $\mathrm{p}$ é o número de níveis de efeitos fixos;

a - vetor contendo os valores genéticos dos animais, de dimensão q x 1;

e - vetor de efeitos ambientais não identificáveis ou erros aleatórios, de dimensão $\mathrm{n} \mathrm{x}$ 1 ;

$\mathrm{X}$ e $\mathrm{Z}$ - são matrizes de delineamento ou de incidência, contendo apenas valores 0 e 1 , as quais associam as observações $(\mathrm{Y})$ aos efeitos fixos $(\beta)$ e aos valores genéticos dos animais (a), respectivamente. $\mathrm{X}$ tem dimensão $(\mathrm{n} \times \mathrm{p})$ e $\mathrm{Z}$ tem dimensão (n x q).

Por este modelo verifica-se que uma observação (Y) em determinado animal, pode ser desdobrada em termos de efeitos ambientais identificáveis $(\beta)$, efeitos genéticos (a) e efeitos ambientais não identificáveis ou aleatórios (e). O objetivo do melhorista de animais é então predizer o valor genético (a) dos animais, a partir de análises estatísticas dos valores medidos (fenotípicos) diretamente nos animais.

Com base neste modelo, os valores genéticos dos animais podem ser preditos resolvendo-se o sistema de equações, denominado equações de modelo misto:

$\left[\begin{array}{l}\beta \\ \hat{a}\end{array}\right]=\left[\begin{array}{cc}X^{\prime} X & X^{\prime} Z \\ Z^{\prime} X & Z^{\prime} Z+A \alpha\end{array}\right]^{-1}\left[\begin{array}{l}X^{\prime} Y \\ Z^{\prime} Y\end{array}\right]$, em que $\mathrm{A}^{-1}$ refere-se à inversa da matriz de parentesco entre os animais e $\alpha=\sigma_{\mathrm{e}}^{2} / \sigma_{\mathrm{a}}^{2}=\left(1-\mathrm{h}^{2}\right) / \mathrm{h}^{2}$, sendo $\sigma_{\mathrm{e}}^{2}$ a variância ambiental, $\sigma_{\mathrm{a}}^{2}$ a variância genética aditiva da característica e $h^{2}$ a herdabilidade da característica.

Dessa forma, tendo-se os dados (Y), conhecendo-se a herdabilidade $\left(\mathrm{h}^{2}\right)$ da característica e preenchendo-se as matrizes $\mathrm{X}, \mathrm{Z}$ e $\mathrm{A}$, pode-se predizer de maneira eficiente, o valor genético (a) 
dos animais pelo modelo animal. A matriz A é obtida diretamente com base na genealogia dos animais, conforme relatado no tópico 6.1 .

Preditos desta maneira, os valores genéticos dos animais são obtidos com a máxima precisão possível, utilizando as informações dos próprios animais e também de seus parentes e considerando também a correção dos dados observados, para os efeitos ambientais identificáveis.

\section{Valores Genéticos Preditos, Diferença Esperada na Progênie, Capacidade Predita de Transmissão e Capacidade Predita de Produção}

Os valores genéticos preditos (â ou EBV) referem-se à avaliação do somatório dos efeitos médios dos alelos que o animal possui (FALCONER, 1989). Uma vez que os animais transmitem à descendência apenas metade de seus valores genéticos, outras quantidades, mais úteis do ponto de vista do criador, foram desenvolvidas pelos pesquisadores, tais como a diferença esperada na progênie (DEP), a capacidade de transmissão (PTA) e a capacidade predita de produção (PPA) (VAN VLECK et al., 1987).

A DEP refere-se à diferença esperada na performance da progênie de um animal em comparação com a performance média das progênies de todos os animais em avaliação. Assim, a DEP equivale à metade do valor genético de um animal, predito como desvio da média geral de todos os animais. A medida denominada DEP é amplamente utilizada nos sumários de avaliação genética em gado de corte, ovinos e suínos (em suínos utiliza-se muito, também, os próprios EBV's).

Em bovinos de leite, uma medida equivalente à DEP, denominada PTA é utilizada nos sumários de Avaliação genética visando a seleção de reprodutores e de matrizes. Entretanto, na seleção de animais (vacas) a permanecer no rebanho a medida de PPA é utilizada, a qual equivale à soma do valor genético predito com o efeito de ambiente permanente associado a determinado animal. Assim, a medida PPA tem uma conexão direta com o parâmetro da repetibilidade.

\section{Acurácia, Confiabilidade e Variância do Erro de Predição}

De maneira geral, os valores genéticos preditos (â ) não são iguais aos valores genéticos verdadeiros (a) dos animais. A proximidade entre estes dois valores pode ser avaliada com base na estatística denominada acurácia (VAN VLECK et al., 1987), a qual refere-se à correlação entre os valores genéticos preditos e os valores genéticos verdadeiros dos animais e é simbolizada por $\boldsymbol{r}_{\text {âa }}$. Em geral, quanto maior a acurácia na avaliação de um animal, maior é a confiança na avaliação e no valor genético predito deste animal.

A distância (â $-\mathbf{a}$ ) entre os valores genéticos preditos e os verdadeiros é função da quantidade $\left(1-r_{\text {âa }}\right)$ e a estatística denominada variância do erro de predição (PEV) dada por $\operatorname{PEV}=\operatorname{Var}(\hat{\mathrm{a}}-\mathrm{a})=\left(1-\mathrm{r}_{\mathrm{a} a \mathrm{a}}^{2}\right) \sigma_{\mathrm{a}}^{2}$

fornece uma medida da precisão na avaliação dos animais. A predição pela metodologia do modelo animal - BLUP é "Best" no sentido de minimizar a $\operatorname{Var}(\hat{a}-a)$ ou seja, maximiza a precisão. A grande utilidade da $\mathrm{PEV}$, entretanto, refere-se à possibilidade de se calcular o desvio (erro) padrão e o intervalo de confiança dos valores genéticos preditos e consequentemente, obter-se uma medida de risco na utilização de determinados animais. Por exemplo, um valor genético predito de $\hat{a}=20 \mathrm{~kg}$ de peso em gado de corte, associado a uma acurácia de $r_{\text {âa }}=0,80$ e uma variância aditiva $\sigma_{a}^{2}=5 \mathrm{~kg}^{2}$, permite a obtenção do seguinte intervalo de confiança (IC) para o valor genético predito:

$$
\mathrm{IC}=\hat{a} \pm t\left[\left(1-r^{2}{ }_{\text {âa }}\right) \sigma^{2}{ }_{a}\right]^{1 / 2}=20 \pm 1,96\left[\left(1-0,8^{2}\right) 5\right]^{1 / 2}=20 \pm 2,63=17,37|------| 22,63
$$

$\mathrm{O}$ valor de $\mathrm{t}$ utilizado $(1,96)$ corresponde ao valor da distribuição $\mathrm{t}$ de Student associado a $95 \%$ de confiança e grande número de graus de liberdade. Assim, para o presente exemplo, pode-se dizer, com $95 \%$ de confiança, que o verdadeiro valor genético (superioridade genética em relação à média do rebanho) do animal está entre $17,37 \mathrm{~kg}$ e $22,63 \mathrm{~kg}$.

A estatística acurácia é muito empregada nos sumários associados à avaliação genética em gado de corte, ovinos e suínos. Em gado de leite é mais utilizada a estatística denominada confiabilidade $\left(r_{\text {âa }}^{2}\right)$ que é, exatamente, o quadrado da acurácia. A confiabilidade é um coeficiente de determinação, ao passo que a acurácia é um coeficiente de correlação. Portanto, a confiabilidade pode ser interpretada como a proporção da variação dos valores preditos (em hipotéticas predições repetidas para o mesmo animal) que pode ser explicada pelo valor genético verdadeiro e a acurácia pode ser interpretada como a correlação entre os ordenamentos ('rankings") dos valores genéticos preditos e dos verdadeiros.

Os sumários de avaliação genética em gado de corte, ovinos e suínos em geral apresentam as 
DEP's (ou EBV's no caso de suínos) e a acurácia na avaliação de cada animal. Em gado de leite, em geral, os sumários apresentam as PTA's e a confiabilidade na avaliação de cada animal. É importante ressaltar que as DEP's (ou PTA's) dos diferentes animais podem ser comparadas diretamente entre si, independentemente da acurácia. As diferentes quantidades e qualidades das informações dos animais já foram consideradas no cálculo da DEP (e também da acurácia). A acurácia simplesmente informa sobre o risco no uso de cada animal e não tem a propriedade de informar se o animal é bom ou não. Esta informação pode ser obtida exclusivamente com base na DEP. Um animal com alta DEP e acurácia mais baixa do que a dos demais, foi mais penalizado na avaliação genética do que os demais animais, ou seja, a média de sua progênie foi mais descontada (forçada ou regredida em direção à média geral) do que as médias das progênies de outros animais. Assim, tal animal, embora apresente um intervalo de confiança mais amplo de seu valor genético predito, com menor limite inferior do intervalo de confiança, apresenta também maior limite superior do intervalo de confiança de seu valor genético predito, e com alguma probabilidade razoável, pode, de fato, ser melhor que os demais animais.

Em conclusão, a acurácia pode ser utilizada como um indicativo da intensidade de utilização dos animais para a reprodução, sendo que os animais com altos valores genéticos preditos e com acurácia mais baixa, devem ser utilizados com ressalvas.

\section{Modelo Animal Aplicado ao Melhoramento de Bovinos de Leite, Bovinos de Corte, Ovinos e Suínos}

Para a plena aplicação do modelo animal ao melhoramento de animais domésticos, torna-se

essencial o conhecimento dos parâmetros genéticos associados aos caracteres de importância econômica, bem como a definição dos efeitos ambientais identificáveis (efeitos fixos) importantes a cada uma das espécies a serem melhoradas.

Bovinos de Leite. Dentre as raças bovinas especializadas na produção de leite destacam-se a Holandesa, a Jersey, a Guernsey e a Pardo Suíça. No Brasil, dentre as raças sob melhoramento genético destacam-se a Gir (LEDIC e TONHATI, 1998; MARTINEZ et al., 1998), a Girolanda (FREITAS, 1998) e a Guzerá, cujos programas de melhoramento são coordenados pela EMBRAPA Gado de Leite.

Dentre os caracteres economicamente importantes para o melhoramento genético do gado leiteiro destacam-se a produção de leite, a produção de gordura, a produção de proteína e a resistência à mastite (baseada na contagem de células somáticas ou CCS).

Os parâmetros genéticos mais relevantes para a avaliação genética em gado leiteiro são a herdabilidade $\left(\mathrm{h}^{2}\right)$ e a repetibilidade (r) visto que os caracteres de interesse podem ser avaliados em várias lactações. As estimativas dos parâmetros a serem utilizados nas equações de modelo misto, devem ser obtidas principalmente pelo método da máxima verossimilhança restrita (REML). Estimativas de $h^{2}$ e $r$, obtidas por REML são apresentadas na Tabela 1 .

Tabela 1. Estimativas de herdabilidade $\left(\mathrm{h}^{2}\right)$ e de repetibilidade (r) em gado bovino leiteiro da raça Holandesa, obtidas pelo método da máxima verossimilhança restrita.

\begin{tabular}{l|c|c|l}
\hline \multicolumn{1}{c|}{ Caráter } & $\mathrm{h}^{2}$ & $\mathrm{r}$ & \multicolumn{1}{c}{ Referência } \\
\hline Produção de leite $(\mathrm{kg})$ & 0,30 & 0,45 & VISSCHER (1991); GODDARD (1992) \\
Produção de gordura (kg) & 0,30 & 0,45 & VISSCHER (1991); GODDARD (1992) \\
Produção de proteína (kg) & 0,30 & - & VISSCHER (1991); GODDARD (1992) \\
Resistência à mastite (CCS) & 0,07 & 0,50 & GADINI et al. (1997) \\
\hline
\end{tabular}

Dentre os efeitos ambientais identificáveis importantes em gado leiteiro, destacam-se: rebanho, ano de parto, estação de parto, freqüência de ordenha, local ou região, grau de sangue e idade da vaca ao parto. Estes efeitos são os efeitos fixos a serem estimados no vetor $\beta$. De maneira geral, os efeitos de rebanho, ano e estação são agrupados em um único efeito (com vários níveis) denominado grupo de manejo.

Bovinos de Corte. Dentre as raças bovinas especializadas na produção de carne destacam-se a
Nelore, a Hereford, a Angus, a Canchim, a Charolesa, dentre outras. No Brasil, as principais raças sob melhoramento são a Nelore (LOBO et al., 1997; SILVA et al., 1997; FERRAZ e ELER, 1998); a Canchim (OLIVEIRA et al., 1998) e as raças Hereford e Angus (CARDELLINO et al., 1998).

Dentre os principais caracteres objetivos do melhoramento genético de bovinos de corte, destacam-se a taxa de desmama, a habilidade materna e o potencial de crescimento dos animais (NOBRE, 1989). A taxa de desmama está 
relacionada à sobrevivência e ao peso da cria até a desmama e a habilidade materna é avaliada com base no peso até a desmama, sendo função da produção de leite das mães. Por outro lado, o potencial de crescimento pode ser inferido com base nas variáveis peso pré-desmama, peso à desmama e peso pós-desmama. Estas avaliações de peso são utilizadas para inferência sobre velocidade de ganho de peso, que também é uma característica essencial em gado de corte, pois está associada à precocidade de crescimento e à precocidade de terminação (FRIES, 1996). Quanto aos caracteres reprodutivos, o mais importante é a precocidade sexual (FRIES, 1996), a qual pode ser avaliada eficientemente pelo perímetro escrotal.

Os parâmetros genéticos mais relevantes para o melhoramento genético de bovinos de corte são a herdabilidade direta $\left(\begin{array}{l}2 \\ \text { D }\end{array}\right)$ e a herdabilidade materna $\left(h_{M}^{2}\right)$, esta última associada a efeitos maternos sobre caracteres de crescimento prédesmama. Estimativas de $\mathrm{h}_{\mathrm{D}}^{2}$ e $\mathrm{h}_{\mathrm{M}}^{2}$, obtidas pelo método REML são apresentadas na Tabela 2.

Tabela 2. Estimativas de herdabilidade direta $\left(h_{D}^{2}\right)$ e herdabilidade materna $\left(h_{M}^{2}\right)$ em gado bovino de corte da raça Nelore, obtidas pelo método da máxima verossimilhança restrita.

\begin{tabular}{|c|c|c|c|}
\hline Caráter & $\mathrm{h}_{\mathrm{D}}^{2}$ & $\mathrm{~h}_{\mathrm{M}}^{2}$ & Referência \\
\hline Peso ao nascer & 0,24 & 0,05 & Mc DONALD (1992) \\
\hline Peso aos 120 dias (kg) & 0,20 & 0,08 & LOBO et al. (1997) \\
\hline Peso aos 240 dias $(\mathrm{kg})$ & 0,23 & 0,08 & LOBO et al. (1997) \\
\hline Peso aos 365 dias $(\mathrm{kg})$ & 0,35 & - & LOBO et al. (1997) \\
\hline Peso aos 550 dias (kg) & 0,36 & - & LOBO et al. (1997) \\
\hline Perímetro escrotal aos 365 dias $(\mathrm{cm})$ & 0,26 & - & LOBO et al. (1997) \\
\hline Perímetro escrotal aos 550 dias (cm) & 0,37 & - & LOBO et al. (1997) \\
\hline
\end{tabular}

Dentre os efeitos ambientais identificáveis importantes em gado de corte, destacam-se: sexo, rebanho, idade, idade da vaca ao parto. De maneira geral, os efeitos de sexo, idade e rebanho são agrupados em um único efeito (com vários níveis) denominado grupo contemporâneo.

Ovinos. Dentre as raças lanadas utilizadas no Brasil destacam-se: Corriedale, Ideal, Merino e Romney Marsh, para a produção de lã e Hampshire Down, Ile de France, Suffolk, Texel, Border Leicester e Poll Dorset para a produção de carne. Programas de melhoramento destas raças vem sendo conduzidos sob a coordenação da ARCO - Associação Brasileira dos Criadores de Ovinos, com sede em Bagé-RS (Benitez, 1996; Cardellino, 1996).

Dentre os principais caracteres objetivos do melhoramento de ovinos, destacam-se a produção (peso) de lã, o diâmetro das fibras da lã, o número de cordeiros nascidos (prolificidade) e o peso corporal. Os pesos corporais pré-desmama permitem a avaliação da habilidade materna (efeito materno) e o próprio potencial de crescimento dos animais. Por outro lado, os pesos pós-desmama refletem apenas os efeitos genéticos diretos para crescimento dos animais.

Os parâmetros genéticos mais relevantes para a avaliação genética em ovinos são a herdabilidade e a repetibilidade para os caracteres produção de lã, diâmetro das fibras e prolificidade e a herdabilidade direta e herdabilidade materna para os caracteres de crescimento pré-desmama. Estimativas de $h^{2}, r, h_{D}^{2}$ e $h_{M}^{2}$, obtidas por REML, são apresentadas na Tabela 3, com base em copilação realizada por RESENDE e ROSA-PEREZ (1998).

Tabela 3. Estimativas de herdabilidade $\left(h^{2}\right)$, repetibilidade $(r)$, herdabilidade direta $\left(h_{D}^{2}\right)$ e herdabilidade materna $\left(h_{M}^{2}\right)$ em ovinos, obtidas pelo método da máxima verossimilhança restrita.

\begin{tabular}{l|c|c|c|c}
\hline \multicolumn{1}{c|}{ Caráter } & $\mathrm{h}^{2}$ & $\mathrm{r}$ & $\mathrm{h}_{\mathrm{D}}^{2}$ & $\mathrm{~h}_{\mathrm{M}}^{2}$ \\
\hline Peso de lã & 0,40 & 0,05 & - & - \\
Diâmetro de fibras & 0,40 & 0,05 & - & - \\
Número de cordeiros nascidos & 0,10 & 0,15 & - & - \\
Peso corporal aos 50 dias & - & - & 0,20 & 0,10 \\
\hline
\end{tabular}


Dentre os efeitos ambientais identificáveis importantes em ovinos, destacam-se os efeitos de grupo contemporâneo, sexo e idade. Estes são os efeitos fixos a serem estimados no vetor $\beta$.

Suínos. Dentre as raças suínas mais utilizadas no Brasil destacam-se a Large White, a Landraee e a Duroc (IRGANG e FAVERO, 1997). Entretanto, a maioria dos animais utilizados são híbridos. Outras raças brasileiras como a Moura, a Piau, a Caruncho e a Canastra são ainda utilizados por pequenos produtores, a nível de subsistência (MARIANTE, 1988).

Dentre os principais caracteres objetivos do melhoramento genético de suínos destacam-se o ganho de peso, a conversão alimentar, a espessura do toucinho, a idade aos $100 \mathrm{~kg}$ e o tamanho da leitegada. Os caracteres ganho de peso e conversão alimentar são altamente correlacionados e a avaliação de apenas um deles pode, em alguns casos, ser suficiente.

Os parâmetros genéticos mais relevantes para o melhoramento genético de suínos são a herdabilidade $\left(\mathrm{h}^{2}\right)$ e a correlação de ambiente comum (efeitos de leitegada) aos indivíduos da família $\left(\mathrm{c}^{2}\right)$. Estimativas de $\mathrm{h}^{2} \mathrm{e}^{2}$, obtidas pelo método REML, são apresentadas na Tabela 4.

Tabela 4. Estimativas de herdabilidade $\left(\mathrm{h}^{2}\right)$ e correlação de ambiente comum aos indivíduos da família $\left(\mathrm{c}^{2}\right)$ em suínos, obtidas pelo método da máxima verossimilhança restrita.

\begin{tabular}{|c|c|c|c|c|}
\hline Raça & Caráter & $\mathrm{h}^{2}$ & $\mathrm{c}^{2}$ & Referência \\
\hline \multirow[t]{3}{*}{ Large White } & Conversão Alimentar & 0,41 & 0,09 & TORRES JÚNIOR et al. (1998) \\
\hline & Espessura do Toucinho & 0,37 & 0,08 & TORRES JÚNIOR et al. (1998) \\
\hline & Idade aos $100 \mathrm{~kg}$ & 0,22 & 0,16 & TORRES JÚNIOR et al. (1998) \\
\hline \multirow[t]{4}{*}{ Landraee } & Conversão Alimentar & 0,34 & 0,07 & TORRES JÚNIOR et al. (1998) \\
\hline & Espessura do Toucinho & 0,51 & 0,06 & TORRES JÚNIOR et al. (1998) \\
\hline & Idade aos $100 \mathrm{~kg}$ & 0,27 & 0,14 & TORRES JÚNIOR et al. (1998) \\
\hline & Tamanho da Leitegada & 0,10 & - & LONG (1992) \\
\hline \multirow[t]{3}{*}{ Duroc } & Ganho de Peso Diário & 0,21 & 0,15 & COSTA et al. (1998); ROSO et al. (1995) \\
\hline & Espessura do Toucinho & 0,31 & 0,09 & COSTA et al. (1998); ROSO et al. (1995) \\
\hline & Peso aos 70 dias & 0,28 & 0,19 & COSTA et al. (1998) \\
\hline
\end{tabular}

Dentre os efeitos ambientais identificáveis importantes em suínos, destaca-se o grupo contemporâneo, que inclui animais de determinado sexo, nascidos em determinado mês em um dado ano.

\section{Exemplos Práticos}

Matriz de Parentesco. A matriz de parentesco A é essencial na avaliação genética pelo BLUP, permitindo a obtenção de resultados mais acurados (pela consideração das informações de parentes) e não viciados (pela consideração da correlação entre os valores genéticos, na estimação dos efeitos fixos).

A matriz A é simétrica e seus elementos da diagonal $\left(\mathrm{a}_{\mathrm{ii}}\right)$ para cada indivíduo í é igual a $\left(1+\mathrm{F}_{\mathrm{i}}\right)$, onde $F_{i}$ é o coeficiente de endogamia do indivíduo i. Os elementos fora $\left(\mathrm{a}_{\mathrm{ij}}\right)$ da diagonal referem-se ao coeficiente de parentesco entre os indivíduos i e j.

Para composição da matriz A, Henderson (1976) descreve os seguintes passos:

- Codificar os animais de 1 a N, ordenados de forma que os parentais precedam suas progênies;

- Se ambos os parentais (s e d) do indivíduo i são conhecidos, fazer:

$\mathrm{a}_{\mathrm{ii}}=1+0,5\left(\mathrm{a}_{\mathrm{sd}}\right)$

$\mathrm{a}_{\mathrm{ji}}=\mathrm{a}_{\mathrm{ij}}=0,5\left(\mathrm{a}_{\mathrm{js}}+\mathrm{a}_{\mathrm{jd}}\right), \mathrm{j}=1 \mathrm{a}(\mathrm{i}-1)$;

- Se somente um parental (s) é conhecido e supostamente não aparentado com a fêmea com que cruzou, fazer:

$\mathrm{a}_{\mathrm{ii}}=1$

$\mathrm{a}_{\mathrm{ji}}=\mathrm{a}_{\mathrm{ij}}=0,5\left(\mathrm{a}_{\mathrm{js}}\right), \mathrm{j}=1 \mathrm{a}(\mathrm{i}-1)$

- Se ambos os parentais são desconhecidos e supostamente não aparentados, fazer:

$\mathrm{a}_{\mathrm{ii}}=1$

$\mathrm{a}_{\mathrm{ji}}=\mathrm{a}_{\mathrm{ij}}=0, \mathrm{j}=1 \mathrm{a}(\mathrm{i}-1)$

Considere-se o seguinte pedigree:

\begin{tabular}{c|c|c}
\hline Indivíduos & Pai & Mãe \\
\hline 4 & 1 & Desconhecida \\
5 & 3 & 2 \\
6 & 1 & 2 \\
7 & 4 & 5 \\
8 & 3 & 6 \\
\hline
\end{tabular}


Para este pedigree, tem-se a seguinte matriz de parentesco:

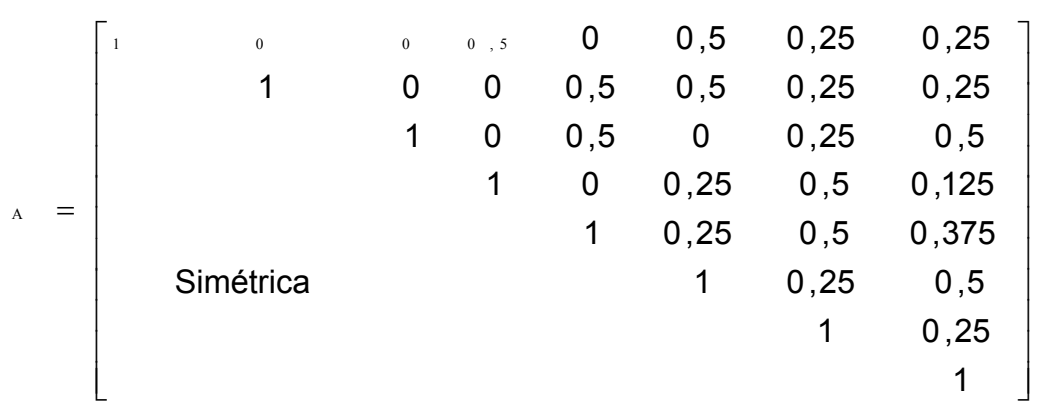

Os elementos foram assim obtidos:

\begin{tabular}{|c|c|}
\hline$a_{11}=1$ & $a_{12}=0$ \\
\hline$a_{22}=1$ & $a_{13}=0$ \\
\hline$a_{33}=1$ & $\mathrm{a}_{14}=0,5\left(\mathrm{a}_{\mathrm{j} s}\right)=0,5\left(\mathrm{a}_{11}\right)=0,5$ \\
\hline$a_{44}=1$ & $a_{15}=0,5\left(a_{j s}+a_{j d}\right)=0,5\left(a_{13}+a_{12}\right)=0,5(0)=0$ \\
\hline$a_{55}=1+0=1$ & $a_{16}=0,5\left(a_{11}+a_{12}\right)=0,5(1+0)=0,5$ \\
\hline$a_{66}=1+0=1$ & $a_{17}=0,5\left(a_{14}+a_{15}\right)=0,5(0,5+0)=0,25$ \\
\hline$a_{77}=1+0=1$ & $a_{18}=0,5\left(a_{13}+a_{16}\right)=0,5(0+0,5)=0,25$ \\
\hline \multicolumn{2}{|l|}{$\mathrm{a}_{88}=1+0=1$} \\
\hline$a_{23}=0$ & $a_{34}=0,5\left(a_{31}\right)=0$ \\
\hline $\mathrm{a}_{24}=0,5\left(\mathrm{a}_{21}\right)=0$ & $a_{35}=0,5\left(a_{33}+a_{32}\right)=0,5$ \\
\hline $\mathrm{a}_{25}=0,5\left(\mathrm{a}_{23}+\mathrm{a}_{22}\right)=0,5(0+1)=0,5$ & $a_{36}=0,5\left(a_{31}+a_{32}\right)=0$ \\
\hline $\mathrm{a}_{26}=0,5\left(\mathrm{a}_{21}+\mathrm{a}_{22}\right)=0,5(1)=0,5$ & $a_{37}=0,5\left(a_{34}+a_{35}\right)=0,25$ \\
\hline $\mathrm{a}_{27}=0,5\left(\mathrm{a}_{24}+\mathrm{a}_{25}\right)=0,5(0,5)=0,25$ & $a_{38}=0,5\left(a_{33}+a_{36}\right)=0,5$ \\
\hline \multicolumn{2}{|l|}{$\mathrm{a}_{28}=0,5\left(\mathrm{a}_{23}+\mathrm{a}_{26}\right)=0,5(0,5)=0,25$} \\
\hline $\mathrm{a}_{45}=0,5\left(\mathrm{a}_{43}+\mathrm{a}_{42}\right)=0$ & $a_{56}=0,5\left(a_{51}+a_{52}\right)=0,25$ \\
\hline $\mathrm{a}_{46}=0,5\left(\mathrm{a}_{41}+\mathrm{a}_{42}\right)=0,25$ & $a_{57}=0,5\left(a_{54}+a_{55}\right)=0,5$ \\
\hline $\mathrm{a}_{47}=0,5\left(\mathrm{a}_{44}+\mathrm{a}_{45}\right)=0,5(0,25+0,25)=0,25$ & $\mathrm{a}_{58}=0,5\left(\mathrm{a}_{53}+\mathrm{a}_{56}\right)=0,5(0,5+0,25)=0,375$ \\
\hline \multicolumn{2}{|l|}{$\mathrm{a}_{48}=0,5\left(\mathrm{a}_{43}+\mathrm{a}_{46}\right)=0,5(0,25)=0,125$} \\
\hline $\mathrm{a}_{67}=0,5\left(\mathrm{a}_{64}+\mathrm{a}_{65}\right)=0,5(0,25+0,25)=0,25$ & $\mathrm{a}_{78}=0,5\left(\mathrm{a}_{73}+\mathrm{a}_{76}\right)=0,5(0,25+0,25)=0,25$ \\
\hline $\mathrm{a}_{68}=0,5\left(\mathrm{a}_{63}+\mathrm{a}_{65}\right)=0,5$ & \\
\hline
\end{tabular}

Como a matriz A é de elevada ordem (NXN), HENDERSON (1976) estabeleceu regras para se escrever diretamente $\mathrm{A}^{-1}$, sem necessidade de inversão. Com parentais não endógamos, os seguintes passos devem ser adotados, partindo-se de uma matriz NXN, completamente zerada:

- Codificar os indivíduos de 1 a N, ordenados de forma que os parentais precedam suas progênies;

- Se ambos os parentais (s e d) do indivíduo são conhecidos adicionar:
2 ao elemento $\mathrm{a}^{\text {ii; }}$

-1 aos elementos $\mathrm{a}^{\text {is }}, \mathrm{a}^{\mathrm{si}}, \mathrm{a}^{\mathrm{id}}$ e $\mathrm{a}^{\mathrm{di}}$;

$1 / 2$ aos elementos $\mathrm{a}^{\mathrm{ss}}, \mathrm{a}^{\mathrm{sd}}, \mathrm{a}^{\mathrm{ds}}$ e $\mathrm{a}^{\mathrm{dd}}$;

- Se apenas um dos parentais (s) do indivíduo i, for conhecido, adicionar:

$4 / 3$ ao elemento $a^{\text {ii; }}$

$-2 / 3$ aos elementos $a^{\text {is }}$ e $a^{\text {si }}$;

$1 / 3$ ao elemento $a^{\text {ss }}$.

- Se ambos parentais do indivíduo i forem desconhecidos, adicionar 1 ao elemento a $^{\text {ii }}$.

Para o presente exemplo, tem-se: 
Predição de Valores Genéticos pelo Modelo Animal BLUP

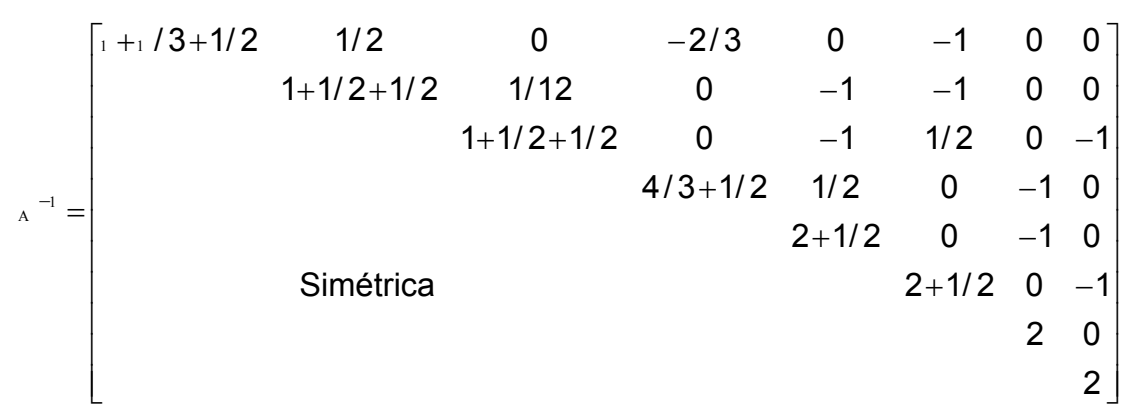

e, portanto:

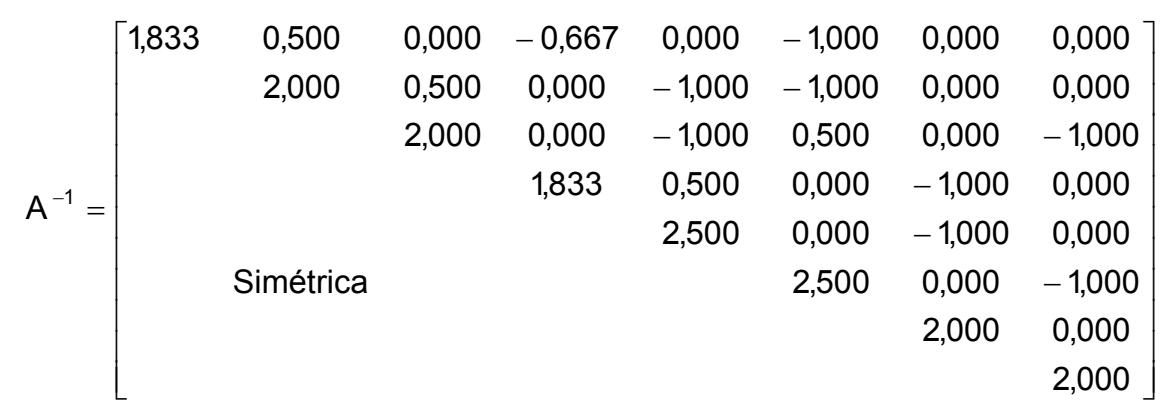

Modelo Animal. Considere-se a avaliação genética de 8 ovinos, classificados por sexo e com as seguintes observações fenotípicas para o caráter peso no final de 12 semanas.

\begin{tabular}{c|c|c|c|c}
\hline Indivíduos & Sexo & Pai & Mãe & Peso $(\mathrm{kg})$ \\
\hline 4 & Macho & 1 & - & $\mathrm{p}_{4}=22,7$ \\
5 & Fêmea & 3 & 2 & $\mathrm{p}_{5}=14,6$ \\
6 & Fêmea & 1 & 2 & $\mathrm{p}_{6}=19,7$ \\
7 & Macho & 4 & 5 & $\mathrm{p}_{7}=17,7$ \\
8 & Macho & 3 & 6 & $\mathrm{p}_{8}=25,3$ \\
\hline
\end{tabular}

O modelo para os dados equivale a $\mathrm{Y}=\mathrm{X} \beta+$ $\mathrm{Z}$ a + e e os efeitos fixos de sexo (masculino-M e feminino-F) e os valores genéticos podem ser obtidos pelas equações de modelo misto: $\left[\begin{array}{l}\hat{\beta} \\ \hat{a}\end{array}\right]=\left[\begin{array}{cc}X^{\prime} X & X^{\prime} Z \\ Z^{\prime} X & Z^{\prime} Z+A^{-1} \alpha\end{array}\right]^{-1}\left[\begin{array}{c}X^{\prime} Y \\ Z^{\prime} Y\end{array}\right]$, em que o símbolo ' significa transposta (por exemplo $\mathrm{X}$ ' é a transposta da matriz X) e -1 indica a matriz inversa.

As matrizes de incidência (que associa os pesos dos animais aos efeitos) para os efeitos fixos (efeitos de sexo) e aleatórios (valores genéticos dos animais) são:

$$
\begin{array}{r}
M \\
X=p_{4} \\
p_{5} \\
p_{6} \\
p_{7}
\end{array}\left[\begin{array}{ll}
1 & 0 \\
0 & 1 \\
p_{8} & 1 \\
1 & 0 \\
1 & 0
\end{array}\right]
$$




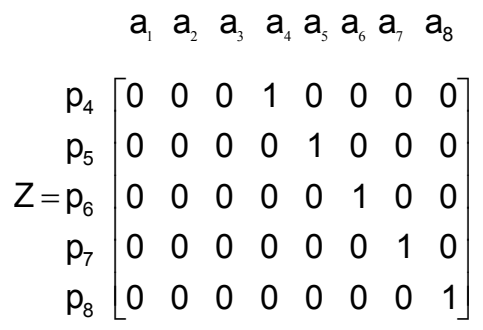

Note-se por Z, que embora os indivíduos 1, 2 e 3 não possuam observação (pesos) pode-se avaliá-los geneticamente com base em informações de seus

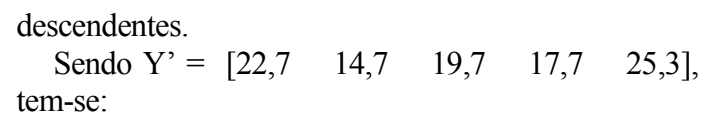

Considerando as relações de parentesco genético aditivo entre os indivíduos em avaliação tem-se que a inversa $\left(\mathrm{A}^{-1}\right)$ da matriz de parentesco é (ver tópico 6.1 em que estas

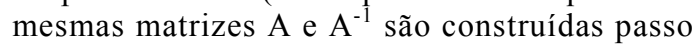
a passo):

$$
A^{-1}\left[\begin{array}{cccccccc}
1.833 & 0.500 & 0.000 & -0.667 & 0.000 & -1.000 & 0.000 & 0.000 \\
0.500 & 2.000 & 0.500 & 0.000 & -1.000 & -1.000 & 0.000 & 0.000 \\
0.000 & 0.500 & 2.000 & 0.000 & -1.000 & 0.500 & 0.000 & -1.000 \\
-0.667 & 0.000 & 0.000 & 1.833 & 0.500 & 0.000 & -1.000 & 0.000 \\
0.000 & -1.000 & -1.000 & 0.500 & 2.500 & 0.000 & -1.000 & 0.000 \\
-1.000 & -1.000 & 0.500 & 0.000 & 0.000 & 2.500 & 0.000 & -1.000 \\
0.000 & 0.000 & 0.000 & -1.000 & -1.000 & 0.000 & 2.000 & 0.000 \\
0.000 & 0.000 & -1.000 & 0.000 & 0.000 & -1.000 & 0.000 & 2.000
\end{array}\right]
$$

Considerando uma herdabilidade média de 0,33 (ASLAMINEJAD e RODEN, 1997) para o caráter peso às 12 semanas em ovinos, tem-se $\alpha=\left(1-h^{2}\right) / h^{2}$ $=2$, de forma que $\mathrm{A}^{-1} \alpha$ é obtida multiplicando-se cada elemento de $A^{-1}$ por 2. Somando $A^{-1} \alpha$ a $Z^{\prime} Z$ e reunindo-se $X^{\prime} X, X^{\prime} Z, Z^{\prime} X$ e $Z^{\prime} Z+A^{-1} \alpha$ em uma única matriz e $X^{\prime} Y$ e $Z$ 'Y em um único vetor, tem-se que as equações de modelo misto são: 
Predição de Valores Genéticos pelo Modelo Animal BLUP

$\left[\begin{array}{l}\hat{\beta}_{1} \\ \hat{\beta}_{2} \\ \hat{a}_{1} \\ \hat{a}_{2} \\ \hat{a}_{3} \\ \hat{a}_{4} \\ \hat{a}_{5} \\ \hat{a}_{6} \\ \hat{a}_{7} \\ \hat{a}_{8}\end{array}\right]=\left[\begin{array}{cccccccccc}3.000 & 0.000 & 0.000 & 0.000 & 0.000 & 1.000 & 0.000 & 0.000 & 1.000 & 1.000 \\ 0.000 & 2.000 & 0.000 & 0.000 & 0.000 & 0.000 & 1.000 & 1.000 & 0.000 & 0.000 \\ 0.000 & 0.000 & 3.667 & 1.000 & 0.000 & -1.333 & 0.000 & -2.000 & 0.000 & 0.000 \\ 0.000 & 0.000 & 1.000 & 4.000 & 1.000 & 0.000 & -2.000 & -2.000 & 0.000 & 0.000 \\ 0.000 & 0.000 & 0.000 & 1.000 & 4.000 & 0.000 & -2.000 & 1.000 & 0.000 & -2.000 \\ 1.000 & 0.000 & -1.333 & 0.000 & 0.000 & 4.667 & 1.000 & 0.000 & -2.000 & 0.000 \\ 0.000 & 1.000 & 0.000 & -2.000 & -2.000 & 1.000 & 6.000 & 0.000 & -2.000 & 0.000 \\ 0.000 & 1.000 & -2.000 & -2.000 & 1.000 & 0.000 & 0.000 & 6.000 & 0.000 & -2.000 \\ 1.000 & 0.000 & 0.000 & 0.000 & 0.000 & -2.000 & -2.000 & 0.000 & 5.000 & 0.000 \\ 1.000 & 0.000 & 0.000 & 0.000 & -2.000 & 0.000 & 0.000 & -2.000 & 0.000 & 5.000\end{array}\right]^{-1}\left[\begin{array}{c}65,7 \\ 34,3 \\ 0 \\ 0 \\ 0 \\ 22,7 \\ 14,6 \\ 19,7 \\ 17,7 \\ 25,3\end{array}\right]$

Resolvendo-se as equações de modelo misto, tem-se:

\begin{tabular}{l|c}
\hline \multicolumn{1}{c|}{ Efeitos } & Soluções \\
\hline Sexo & 22,0274 \\
$\hat{\beta}_{1}$ (Masculino) & 17,1677 \\
$\hat{\beta}_{2}$ (Feminino) & \\
Animais (Valores genéticos) & 0,4979 \\
$\hat{a}_{1}$ & $-0,0908$ \\
$\hat{a}_{2}$ & $-0,2057$ \\
$\hat{a}_{3}$ & $-0,0535$ \\
$\hat{a}_{4}$ & $-0,9386$ \\
$\hat{a}_{5}$ & $-0,9032$ \\
$\hat{a}_{6}$ & $-1,2623$ \\
$\hat{a}_{7}$ & 0,9335 \\
$\hat{a}_{8}$ & \\
\hline
\end{tabular}

Com base nestes resultados pode-se concluir, por exemplo, que os animais machos tem peso médio de $22,0274 \mathrm{~kg}$ e as fềmeas de $17,1677 \mathrm{~kg}$. Pode-se inferir também que o animal 1 tem valor genético de $0,4979 \mathrm{~kg}$ acima da média geral dos pesos e o animal 7 tem valor genético de $1,2623 \mathrm{~kg}$ abaixo da média geral.

As soluções para as equações de modelo misto podem ser facilmente obtidas pelo software MATLAB (MATLAB, 1992), através do seguinte procedimento:

(1) Digitar os dados no vetor $Y$

$>\mathrm{Y}=\left[\begin{array}{lllll}22,7 & 14,6 & 19,7 & 17,7 & 25,3\end{array}\right]^{\prime}$

(Enter)

(2) Digitar a matriz $X$

$>\mathrm{X}=\left[\begin{array}{llllllllll}1 & 0 & 0 & 1 & 1 ; & 0 & 1 & 1 & 0 & 0\end{array}\right]^{\prime}$

(Enter)

(3) Digitar a matriz $Z$

$>\mathrm{Z}=\left[\begin{array}{lllllllllllll}0 & 0 & 0 & 1 & 0 & 0 & 0 & 0 ; & 0 & 0 & 0 & 0 & 1\end{array}\right.$

$\begin{array}{lllllllllllllll}0 & 0 & 0 ; & 0 & 0 & 0 & 0 & 0 & 1 & 0 & 0 ; & 0 & 0 & 0 & 0\end{array}$

$\begin{array}{llllllllllll}0 & 0 & 1 & 0 ; & 0 & 0 & 0 & 0 & 0 & 0 & 0 & 1]\end{array}$

(Enter)
(4) Digitar a matriz A (do tópico 6.1) de maneira similar à realizada para a matriz $\mathrm{Z}$

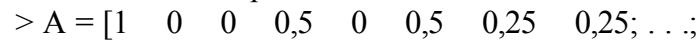
$\left.\begin{array}{llllllll}0,25 & 0,25 & 0,5 & 0,125 & 0,375 & 0,5 & 0,25 & 1\end{array}\right]$ (Enter)

(5) Obter a "matriz dos coeficientes" das equações de modelo misto dada por $M C=\left[\begin{array}{cc}X^{\prime} X & X^{\prime} Z \\ Z^{\prime} X & Z^{\prime} Z+A^{-1} \alpha\end{array}\right]$

$>\mathrm{MC}=\left[\mathrm{X}^{\prime} * \mathrm{X} \quad \mathrm{X}^{\prime} * \mathrm{Z} ; \quad \mathrm{Z}^{\prime} * \mathrm{X} \quad \mathrm{Z}^{\prime} * \mathrm{Z}+\operatorname{inv}(\mathrm{A}) * 2\right]$

(Enter)

(6) Obter o vetor $D=\left[\begin{array}{l}X^{\prime} Y \\ Z^{\prime} Y\end{array}\right]$

$>D=\left[X^{\prime} * Y ; \quad Z^{\prime} * Y\right]$

(Enter)

(7) Obter os resultados finais

$\mathrm{R}=\mathrm{inv}(\mathrm{MC}) * \mathrm{D}$

(Enter) 
A acurácia na avaliação de cada animal pode ser obtida a partir da inversa da matriz dos coeficientes dada por:

$\begin{array}{cc}C^{11} & C^{12}{ }_{\text {ù }} \\ { }_{\tilde{e}}^{*} C^{21} & C^{22 \hat{u}}{ }^{\hat{u}}\end{array}=\left[\begin{array}{cc}X^{\prime} X & X^{\prime} Z \\ Z^{\prime} X & Z^{\prime} Z+A^{-1} \alpha\end{array}\right]^{-1}$

A partir desta matriz tem-se as seguintes igualdades:

$\operatorname{Diag}\left(C^{22}\right) \sigma_{e}^{2}=P E V_{i}=J \sigma_{a}^{2}-r_{\hat{a}_{i}}^{2} \sigma_{a}^{2}, \mathrm{em}$ que diag $\left(\mathrm{C}^{22}\right)$ equivale à diagonal da matriz $\mathrm{C}^{22}, \mathrm{~J}$ referese a um vetor de uns e $r_{\hat{a} a_{i}}$ ao vetor de acurácias.

Assim, a acurácia na avaliação de cada indivíduo (i) pode ser obtida através da expressão $r_{\hat{a ̂ a}_{\mathrm{i}}}=\left[1-\mathrm{PEV} V_{\mathrm{i}} / \sigma_{\mathrm{a}}^{2}\right]^{1 / 2}$, onde $\mathrm{PEV}_{\mathrm{i}}$ é um vetor com os elementos da diagonal de $\mathrm{C}^{22}$, correspondentes a cada animal i.

No presente exemplo e considerando $\sigma_{a}^{2}=2,75$ e $\sigma_{e}^{2}=5,5$ tem-se que:

\begin{tabular}{c|c|c|c}
\hline Animal & $\operatorname{Diag}\left(\mathrm{C}^{22}\right)$ & $\mathrm{PEV}_{\mathrm{i}}$ & $\mathrm{r}_{\text {âa }}$ \\
\hline 1 & 0,471 & 2,5905 & 0,241 \\
2 & 0,492 & 2,7060 & 0,126 \\
3 & 0,456 & 2,5080 & 0,297 \\
4 & 0,428 & 2,3540 & 0,379 \\
5 & 0,428 & 2,3540 & 0,379 \\
6 & 0,442 & 2,4310 & 0,341 \\
7 & 0,442 & 2,4310 & 0,341 \\
8 & 0,422 & 2,4310 & 0,395 \\
\hline
\end{tabular}

Empregando o software MATLAB as acurácias do presente exemplo podem ser obtidas pelo seguinte procedimento:

Enter)

$$
>\mathrm{PEV}_{\mathrm{i}}=\operatorname{diag}(\operatorname{inv}(\mathrm{MC})) * 5,5
$$

$>\mathrm{J}=$ ones $(10,1)$

(Enter)

$$
>r_{\hat{a ̂ a}_{i}}=\operatorname{sqrt}\left(J-P E V_{i} / 2,75\right)
$$

(Enter)

\section{Modelos Animais mais Complexos}

Modelo Animal com Efeito de Ambiente Permanente (Modelo de Repetibilidade).O modelo de repetibilidade (muito utilizado no melhoramento de gado de leite) é dado por:

$\mathrm{Y}=\mathrm{X} \beta+\mathrm{Za}+\mathrm{Wp}+\mathrm{e}$, onde:

$\mathrm{Y}$ - vetor das observações; $\beta$ - vetor de efeitos fixos;

a - vetor de valores genéticos aditivos, aleatório; $\mathrm{p}$ - vetor de efeitos permanentes, aleatório;

e - vetor de efeitos ambientais, aleatório.

$\mathrm{X}, \mathrm{Z}$ e W são matrizes de incidência para os referidos efeitos.

$\mathrm{O}$ vetor $\mathrm{p}$ de efeitos permanentes inclui os efeitos de ambiente permanente e os efeitos não aditivos. Tem-se a seguinte estrutura de variâncias:

$\operatorname{Var}(p)=I \sigma_{p}^{2}$, onde $\sigma_{p}^{2}$ é a variância dos efeitos permanentes

$$
\begin{aligned}
& \operatorname{Var}(\mathrm{e})=\mathrm{I} \sigma_{\mathrm{e}}^{2}=\mathrm{R} \\
& \operatorname{Var}(\mathrm{a})=\mathrm{A} \sigma_{\mathrm{a}}^{2} \\
& \operatorname{Var}(\mathrm{Y})=\mathrm{ZAZ} \mathrm{Z}^{\prime} \sigma_{\mathrm{a}}^{2}+\mathrm{WI} \sigma_{\mathrm{p}}^{2} \mathrm{~W}^{\prime}+\mathrm{R}
\end{aligned}
$$

As equações de modelo misto para o presente caso são:

$$
\left[\begin{array}{l}
\hat{\beta} \\
\hat{a} \\
\hat{p}
\end{array}\right]\left[\begin{array}{ccc}
X^{\prime} X & X^{\prime} Z & X^{\prime} W \\
Z^{\prime} X & Z^{\prime} Z+A^{-1} \alpha_{1} & Z^{\prime} W \\
W^{\prime} X & W^{\prime} Z & W^{\prime} W+I \alpha_{2}
\end{array}\right]=\left[\begin{array}{c}
X^{\prime} Y \\
Z^{\prime} Y \\
W^{\prime} Y
\end{array}\right] \text {, onde }
$$

$\alpha_{1}=\sigma_{\mathrm{e}}^{2} / \sigma_{\mathrm{a}}^{2}=(1-\mathrm{r}) / \mathrm{h}^{2}$

$\alpha_{2}=\sigma_{\mathrm{e}}^{2} / \sigma_{\mathrm{p}}^{2}=(1-r) /\left(r-\mathrm{h}^{2}\right)$

$\sigma_{p}^{2}=$ variância dos efeitos permanentes. 
Modelo Animal com Efeito de Ambiente Comum. Além dos efeitos genéticos, ambientais permanentes e ambientais temporários, efeitos ambientais comuns a determinado grupo de indivíduos parentes, podem contribuir para os valores fenotípicos. Neste caso, a semelhança (covariância) entre parentes não é função apenas da variação genética, mas também da variação devida ao ambiente comum. Assim, a covariância entre indivíduos dentro de família aumenta, fato que conduz a um aumento da variação entre famílias, na quantidade $\sigma_{\mathrm{c}}^{2}$, onde $\sigma_{\mathrm{c}}^{2}$ é a variância de ambiente

Tem-se a seguinte estrutura de variâncias: comum. A inclusão destes efeitos na avaliação genética animal, torna-se relevante quando são avaliadas famílias de irmãos germanos ou de meios irmãos maternais.

O modelo linear com efeitos ambientais comuns (muito empregado no melhoramento de suínos) é dado por:

$\mathrm{Y}=\mathrm{X} \beta+\mathrm{Za}+\mathrm{Wc}+\mathrm{e}$, que é essencialmente, $\mathrm{o}$ modelo de repetibilidade, porém, substituindo o vetor de efeitos permanentes, pelo vetor c, de efeitos ambientais comuns. W neste caso, associa o vetor de dados ao vetor $\mathrm{c}$, ou seja, às famílias.

$$
\begin{aligned}
& \operatorname{Var}(\mathrm{c})=\mathrm{I} \sigma_{\mathrm{c}}^{2} \\
& \operatorname{Var}(\mathrm{e})=\mathrm{I} \sigma_{\mathrm{e}}^{2} \\
& \operatorname{Var}(\mathrm{a})=\mathrm{A} \sigma_{\mathrm{a}}^{2} \\
& \operatorname{Var}(\mathrm{Y})=\mathrm{ZAZ} \mathrm{Z}_{\mathrm{a}}^{2}+\mathrm{WI} \sigma_{\mathrm{c}}^{2} \mathrm{~W}^{\prime}+\mathrm{R}
\end{aligned}
$$

As equações de modelo misto são:

$$
\begin{aligned}
& {\left[\begin{array}{l}
\hat{\beta} \\
\hat{a} \\
\hat{c}
\end{array}\right]\left[\begin{array}{ccc}
X^{\prime} X & X^{\prime} Z & X^{\prime} W \\
Z^{\prime} X & Z^{\prime} Z+A^{-1} \alpha_{1} & Z^{\prime} W \\
W^{\prime} X & W^{\prime} Z & W^{\prime} W+l \alpha_{2}
\end{array}\right]=\left[\begin{array}{c}
X^{\prime} Y \\
Z^{\prime} Y \\
W^{\prime} Y
\end{array}\right] \text {, com }} \\
& \alpha_{1}=\sigma_{e}^{2} / \sigma_{a}^{2}=\left(1-h^{2}-c^{2}\right) / h^{2} \\
& \alpha_{2}=\sigma_{e}^{2} / \sigma_{c}^{2}=\left(1-h^{2}-c^{2}\right) / c^{2} \\
& \sigma_{c}^{2}=c^{2} \sigma_{F}^{2} \\
& c^{2}=\text { correlação de ambiente comum. } \\
& \sigma_{F}^{2}=\text { variância fenotípica. }
\end{aligned}
$$

Modelo Animal com Efeito Materno. A covariância ambiental entre irmãos germanos ou meios irmãos maternos pode ser também devida à habilidade materna ou efeitos maternos, em adição aos efeitos ambientais comuns. A variação na habilidade materna das fêmeas tem uma base genética, e é resultante da variação genética para alguns caracteres das mães, tais quais produção de leite, dentre outros. Caracteres tais como peso pré-desmama, são influenciados por efeitos genéticos diretos, devido aos genes dos próprios indivíduos e também por efeitos genéticos maternos, de outros caracteres das mães. Assim, a capacidade da fêmea propiciar um ambiente favorável para a expressão de caracteres na progênie é parcialmente genético e parcialmente ambiental.

$\mathrm{O}$ modelo linear adequado à descrição de caracteres com efeito materno (amplamente utilizado em gado de corte) equivale a:

$\mathrm{Y}=\mathrm{X} \beta+\mathrm{Za}+\mathrm{Wm}+\mathrm{Sp}+\mathrm{e}$, onde:

$\mathrm{Y}$ - vetor dos dados;

$\beta-$ vetor dos efeitos fixos;

a - vetor de valores genéticos aditivos diretos, aleatório;

$\mathrm{m}$ - vetor de efeitos genéticos maternais (indiretos), aleatório;

$\mathrm{p}$ - vetor aleatório de efeitos permanentes, incluindo influências ambientais permanentes e efeitos genéticos não aditivos maternais da fêmea;

$\mathrm{e}-$ vetor de efeitos residuais, aleatório. 
Para o modelo, tem-se a seguinte estrutura de variâncias:

$$
\begin{aligned}
& \operatorname{Var}\left[\begin{array}{l}
\mathrm{a} \\
\mathrm{m} \\
\mathrm{p} \\
\mathrm{e}
\end{array}\right]=\left[\begin{array}{cccccc}
\mathrm{A} & \sigma_{\mathrm{a}}^{2} & \mathrm{~A} & \sigma_{\mathrm{a}, \mathrm{am}} & 0 & 0 \\
\mathrm{~A} & \sigma_{\mathrm{a}, \mathrm{am}} & \mathrm{A} & \sigma_{\mathrm{am}}^{2} & 0 & 0 \\
0 & 0 & \mathrm{I} \sigma_{\mathrm{p}}^{2} & 0 \\
0 & 0 & 0 & \mathrm{I} \sigma_{\mathrm{e}}^{2}
\end{array}\right] \text {, onde: } \\
& \sigma_{\mathrm{a}}^{2}-\text { variância aditiva para os efeitos diretos; } \\
& \sigma_{\mathrm{am}}^{2} \text { - variância aditiva para os efeitos maternos; } \\
& \sigma_{\mathrm{a}, \mathrm{am}}-\text { covariância aditiva entre efeitos genéticos diretos e maternais; } \\
& \sigma_{p}^{2}-\text { variância dos efeitos permanentes; } \\
& \sigma_{\mathrm{e}}^{2} \text { - variância residual. }
\end{aligned}
$$

Tem-se ainda:

$$
\operatorname{Var}(y)=\left[\begin{array}{ll}
Z & W
\end{array}\right]\left[\begin{array}{cccc}
A & \sigma_{a}^{2} & A & \sigma_{a, a m} \\
A & \sigma_{a, a m} & A & \sigma_{a m}^{2}
\end{array}\right]\left[\begin{array}{c}
Z^{\prime} \\
W^{\prime}
\end{array}\right]+S I \sigma_{p}^{2} S^{\prime}+I \sigma_{e}^{2}
$$

As equações de modelo misto equivalem a:

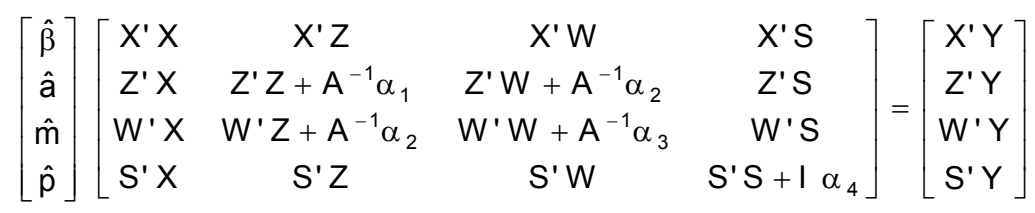

com:

$$
\mathbf{G}=\left[\begin{array}{cc}
\sigma_{\mathrm{a}}^{2} & \sigma_{\mathrm{a}, \mathrm{am}} \\
\sigma_{\mathrm{a}, \mathrm{am}} & \sigma_{\mathrm{am}}^{2}
\end{array}\right] ; \quad \mathrm{G}^{-1}=\left[\begin{array}{ll}
\mathrm{a}^{11} & \mathrm{a}^{12} \\
\mathrm{a}^{21} & \mathrm{a}^{22}
\end{array}\right] ;\left[\begin{array}{ll}
\alpha_{1} & \alpha_{2} \\
\alpha_{2} & \alpha_{3}
\end{array}\right]=\sigma_{\mathrm{e}}^{2}\left[\begin{array}{cc}
a^{11} & a^{12} \\
a^{21} & a^{22}
\end{array}\right] \text { e } \alpha_{4}=\sigma_{\mathrm{e}}^{2} / \sigma_{\mathrm{p}}^{2}
$$

A matriz $\mathrm{G}$ é uma matriz de covariância genética entre os dois caracteres, que são distintos nas mães, mas que afetam um mesmo caráter na progênie. A correlação entre os dois caracteres é dada por $r_{\mathrm{a}, \mathrm{am}}=\sigma_{\mathrm{a}, \mathrm{am}} /\left(\sigma_{\mathrm{a}} \sigma_{\mathrm{am}}\right) \mathrm{e}$ pode ser negativa indicando antagonismo entre os dois caracteres.

Exemplos referentes a estes modelos mais complexos são apresentados detalhadamente por RESENDE e FREITAS (1999).

\section{REFERÊNCIAS BIBLIOGRÁFICAS}

ASLAMINEJAD, A.A.; RODEN, J.A. Estimation of direct and maternal genetic parameters for 12 week weight for Welsh Moutain Sheep. Proceedings of the British Society of Animal Production. 53:177, 1997.

BENITEZ, D. Avaliações genéticas de reprodutores ovinos coordenadas pela Associação Brasileira de Criadores de Ovinos. In: BRUM, A. (ed.). XIV Congresso Estadual de Zootecnia. Anais. PUC-RS. p.187-200, 1996.

CARDELLINO, R.A. Perspectivas para o melhoramento genético ovino. In: BRUM, A. (ed.). XIV Congresso Estadual de Zootecnia. Anais. PUC-RS. p.181-186, 1996.
CARDELLINO, R.A.; CARDOSO, F.F.; CAMPOS, L.T. DEP na origem de touros Angus e Hereford importados dos EUA. Simpósio Nacional da Sociedade Brasileira de Melhoramento Animal, 2, Uberaba, p.339-340, 1998.

COSTA, A.R.C.; LOPES, P.S.; TORRES, R.A.; EUCLYDES, R.F. Estimação de parâmetros genéticos em características de desempenho de suínos da raça Duroc. Reunião Anual da Sociedade Brasileira de Zootecnia, 35. Botucatu. Anais, p.383-385, 1998.

FALCONER, D.S. Introduction to quantitative genetics. $3^{\text {rd }}$. Edn. Longman, Harlow. 438p, 1989.

FERRAZ, J.B.S.; ELLER, J.P. Qualidade dos dados coletados. Simpósio Nacional da Sociedade Brasileira de Melhoramento Animal, 2. Uberaba. Anais, p.265-269, 1998.

FREITAS, A.F. Teste de progênie na raça Girolanda. Simpósio Nacional da Sociedade Brasileira de Melhoramento Animal, 2. Uberaba. Anais, p.417419, 1998.

FRIES, L.A. Precocidade, precocidade e precocidade. Reunião da ABCZ. Uberaba. Anais, 1996.

GADINI, C.H.; KEOWN, J.F.; VAN VLECK, L.D. Parâmetros genéticos do escore de células somáticas. Reunião Anual da Sociedade Brasileira de Zootecnia, 34. Juiz de Fora. Anais. 3:41-43, 1997. 
Predição de Valores Genéticos pelo Modelo Animal BLUP

GODDARD, M.E. Genetic evaluation in the dairy industry. In: HAMMOND, K.; GRASER, H.U.; Mc DONALD, C.A. Animal Breeding: The Modern Approach. Post Graduate Foundation of Veterinary Science. Sydney, p.95-102, 1992.

HENDERSON, C.R. Sire evaluation and genetic trends. In: Animal Breeding and Genetics Symposium in Honor of J. Lush. American Society of Annimal Science, Champaign, III, p.10-41, 1973.

HENDERSON, C.R. A simple method for computing the inverse of a numerator relationship matrix used in prediction of breeding values. Biometrics, 32:69-83, 1976.

HENDERSON, C.R. Aplications of linear models in animal breeding. University of Guelph, Guelph. 462p, 1984.

IRGANG, R.; FAVERO, J.A. Reprodutores suínos de alto valor genético para número de leitões nascidos por leitegada. EMBRAPA-CNPSA. Concórdia. 76p. (Documentos, 43), 1997.

LEDIC, I.L.; TONHATI, H. O impacto mercadológico da implantação do Programa Nacional de Melhoramento do Gir Leiteiro. Simpósio Nacional da Sociedade Brasileira de Melhoramento Animal, 2. Uberaba. Anais, p.407-409, 1998.

LOBO, R.B.; REYES, A. de Los; BEZERRA, L.A.F. Avaliação genética de animais jovens, touros e matrizes. Programa de Melhoramento Genético da Raça Nelore. Ribeirão Preto, Depto. de Genética FMRP-USP, 72p, 1997.

LONG, T. Genetic evaluation in the pig industry. In: HAMMOND, K.; GRASER, H.U.; Mc DONALD, C.A. Animal breeding. The modern approach. Sydney: University of Sydney. p.103-110, 1992.

MARIANTE, A.S. Conservação de germoplasma animal no Brasil. In: ARAÚJO, S.M.C.; OSUNA, J.A. (eds.). Encontro sobre recursos genéticos. Jaboticabal. FCAVJ/UNESP, p.148-161, 1988.

MARTINEZ, M.L.; VERNEQUE, R.S.; TEODORO, R.L. Comparação de procedimentos de avaliação genética em vacas da raça Gir. Reunião Anual da Sociedade Brasileira de Zootecnia, 35. Botucatu, 1998. Anais, v.3, p.237-239, 1998.

MATLAB - High-performance numeric computation and visualization software - Reference Guide. Natick, MA: The Math Works, Inc., 1992.
Mc DONALD, C.A. Genetic evaluation of beef cattle. . In: HAMMOND, K.; GRASER, H.U.; Mc DONALD, C.A. Animal breeding. The modern approach. Sydney: University of Sydney. p.77-84, 1992.

NOBRE, P.R.C. Avaliação de reprodutores em bovinos de corte. Campo Grande, EMBRAPA-CNPGC. 27p. (EMBRAPA-CNPGC. Documentos, 42), 1989.

OLIVEIRA, H.N.; LOBO, R.B.; MEDEIROS, G.; BEZERRA, L.A.F.; CORRADO, M.P. Programa Nacional de Melhoramento Genético da Raça Canchim. Simpósio Nacional da Sociedade Brasileira de Melhoramento Animal, 2. Uberaba. Anais, p.369370, 1998.

RESENDE, M.D.V.; FREITAS, A.F. Genética Quantitativa e Estatística no Melhoramento Animal. EMBRAPA-CPPSE. São Carlos. 465p, 1999.

RESENDE, M.D.V.; ROSA-PEREZ, J.R.H. Genética e Melhoramento de Ovinos. Curitiba: Editora da UFPR. 68p, 1998.

ROSO, V.M.; FRIES, L.A.; MARTINS, E.S. Parâmetros genéticos em características de desempenho e qualidade de carcaça em suínos da raça Duroc. Revista da Sociedade Brasileira de Zootecnia. 24:p.310-316, 1995.

SILVA, L.O.C.; EUCLIDES FILHO, K.; NOBRE, P.R.C.; JOSAHKIAN, L.A. Tendências genéticas na raça Nelore no Brasil. Reunião Anual da Sociedade Brasileira de Zootecnia, 34. Juiz de Fora. Anais, p.175-176, 1997.

TORRES JUNIOR, R.A.A.; SILVA, M.A.; LOPES, P.S.; REGAZZI, A.J.; EUCLIDES, R.F. Estimativas de componentes de covariância para características produtivas de suínos Landrace e Large White pelo método da máxima verossimilhança restrita. Revista da Sociedade Brasileira de Zootecnia. 27(2):283291, 1998.

VAN VLECK, L.D.; POLLAK, E.J.; OLTENACU, E.A.B. Genetics for the animal sciences. W.H. Freeman, New York. 391p, 1987.

VISSCHER, P.M. Estimation of genetic parameters in dairy cattle using an animal model and implications for genetic evaluation. $\mathrm{PhD}$ Thesis, University of Edinburgh, Edinburgh, U.K. 1991. 